\title{
ON INNER AUTOMORPHISMS OF FINITE GROUPS
}

\author{
MARTIN R. PETTET
}

(Communicated by Warren J. Wong)

\begin{abstract}
It is shown that in certain classes of finite groups, inner automorphisms are characterized by an extension property and also by a dual lifting property. This is a consequence of the fact that for any finite group $G$ and any prime $p$, there is a $p$-group $P$ and a semidirect product $H=G P$ such that $P$ is characteristic in $H$ and every automorphism of $H$ induces an inner automorphism on $H / P$.
\end{abstract}

Every inner automorphism of a group $G$ possesses the property that it extends to an automorphism of any group which contains $G$ as a subgroup. P. Schupp [2] has shown that in the category of all groups, this extendibility property characterizes inner automorphisms. However, since Schupp's argument uses a free product construction, the question arises whether inner automorphisms can be similarly characterized in classes of groups which are not closed under free products.

In this note, we confirm that the extendibility property does indeed characterize inner automorphisms in certain classes of finite groups. As it happens, with the same stroke the dual question of whether inner automorphisms are precisely those which can be lifted to homomorphic pre-images is also settled (affirmatively) for those classes. The basis for these conclusions amounts to an observation about a remarkable construction of $\mathrm{H}$. Heineken and $\mathrm{H}$. Liebeck [1] (and a subsequent extension of U. H. M. Webb [3]).

Theorem. Let $G$ be a finite group and let $p$ be any prime. Then there exists a finite special p-group $P$ on which $G$ acts faithfully such that $P$ is a characteristic subgroup of the corresponding semidirect product $H=G P$, and such that every automorphism of $H$ induces on $H / P$ an inner automorphism.

Corollary. Let $\pi$ be any set of primes and let $\Gamma$ be the class of all finite $\pi$ groups, the class of all finite solvable $\pi$-groups, or the class of all finite nilpotent $\pi$-groups. Suppose that $G \in \Gamma$ and that $\alpha$ is an automorphism of $G$. Then the following statements are equivalent:

(i) $\alpha$ is an inner automorphism of $G$.

Received by the editors September 14, 1988 and, in revised form, November 16, 1988. 1980 Mathematics Subject Classification (1985 Revision). Primary 20D45. 
(ii) For every $H \in \Gamma$ and every epimorphism $\eta: H \rightarrow G$, there exists an automorphism $\hat{\alpha}$ of $H$ such that

$$
\hat{\alpha} \eta=\eta \alpha .
$$

(That is, $\alpha$ lifts to every homomorphic pre-image of $G$ in $\Gamma$.)

(iii) For every $H \in \Gamma$ and every monomorphism $\theta: G \rightarrow H$, there exists an automorphism $\tilde{\alpha}$ of $H$ such that

$$
\theta \tilde{\alpha}=\alpha \theta \text {. }
$$

(That is, $\alpha$ extends to every group in $\Gamma$ in which $G$ is embedded.)

\section{The CONSTRuCtions OF HeineKen-Liebeck AND WebB}

Suppose first that $p$ is an odd prime. To each directed graph $D$ with vertex set $\left\{V_{1}, V_{2}, \ldots, V_{n}\right\}$, Heineken and Liebeck [1] associate a special $p$-group with canonical generators $x_{1}, x_{2}, \ldots, x_{n}$. The center of $P$ is elementary abelian with basis $\left\{\left[x_{r}, x_{s}\right]: 1 \leq r<s \leq n\right\}$ and in addition, if $y_{i}$ is the product (in any order) of the $x_{j}$ 's corresponding to those vertices $V_{j}$ for which there is an edge from $V_{i}$ to $V_{j}$, then $P$ satisfies the relations

$$
x_{i}^{p}=\left[x_{i}, y_{i}\right], \quad 1 \leq i \leq n .
$$

Aut $D$ may then be identified as a subgroup of Aut $P$ which faithfully permutes the $x_{i}$ 's (and, in fact, $P / Z(P)$ is the corresponding permutation module). The bulk of [1] is devoted to proving that if $D$ is strongly connected and satisfies certain additional conditions, then Aut $P$ is the semidirect product of Aut $D$ with the subgroup $C_{\mathrm{Aut} P}(P / Z(P))$ of central automorphisms.

By a much shorter argument, Heineken and Liebeck also show that, given a set of generators for a finite group $G$, one may construct a strongly connected directed graph $D$ (satisfying the "additional conditions" alluded to above) with the property that $G \cong$ Aut $D$. If $|G| \leq 4$, the construction is ad hoc [1, p. 15] but otherwise, the graph $D$ is a variation on the Cayley diagram. Here, it suffices simply to record the fact that $D$ has no closed paths of length less than 3 (and in particular, since it is strongly connected, $D$ has at least three vertices).

The upshot of these results is that, given any finite group $G$ and any odd prime $p$, there exists a finite special $p$-group $P$ for which Aut $P$ is the semidirect product of $C_{\text {Aut } P}(P / Z(P))$ with a subgroup isomorphic to $G$.

We need one further observation. Suppose that $P$ is the $p$-group constructed as described above from the directed graph $D$ associated with a finite group $G$. If $x$ is any element of $P$, we may write

$$
x=x_{1}^{a_{1}} x_{2}^{a_{2}} \cdots x_{n}^{a_{n}} z
$$

where $0 \leq a_{i}<p$ for every $i$ and $z \in Z(P)$. If $x$ has order $p$, then

$$
\begin{aligned}
1 & =x_{1}^{p a_{1}} x_{2}^{p a_{2}} \cdots x_{n}^{p a_{n}} \\
& =\left[x_{1}, y_{1}\right]^{a_{1}}\left[x_{2}, y_{2}\right]^{a_{2}} \cdots\left[x_{n}, y_{n}\right]^{a_{n}} .
\end{aligned}
$$


(The first equality depends, of course, on the fact that $p \neq 2$.) Since $D$ is strongly connected and $n>2$, we may choose, for each vertex $V_{i}$, another vertex $V_{j}$ such that $D$ contains an edge from $V_{i}$ to $V_{j}$. Then $x_{j}$ appears as a "factor" in $y_{i}$ and so, in the expansion of $\left[x_{i}, y_{i}\right]^{a_{i}}$ with respect to the basis $\left\{\left[x_{r}, x_{s}\right]: 1 \leq r<s \leq n\right\}$, we find the term $\left[x_{i}, x_{j}\right]^{a_{i}}$. But $D$ has no closed paths of length 2 and so $x_{i}$ is not a "factor" of $y_{j}$. We conclude, therefore, that $a_{i}=0$ for every $i$, whence $x=z \in Z(P)$. Thus, $Z(P)=\Omega_{1}(P)$.

Webb's paper [3] is an extension of the Heineken-Liebeck work (using undirected graphs) and in particular, treats the case that $p=2$. (See Theorems 3.2, 5.8 , and the concluding paragraph.) Most of the remarks above remain valid, including the fact that the number of vertices of the graph (and hence, the number of generators of the associated $p$-group $P$ ) is at least 3. Also, if $p=2$, $Z(P)=\Omega_{1}(P)$ [3, Lemma 3.1 (iv)]. (This last equality does not apply to Webb's construction when $p$ is odd, which is why in this case, the Heineken-Liebeck construction is more convenient for our purposes.)

\section{PROOF OF THE THEOREM}

Using the Heineken-Liebeck recipe if $p$ is odd and the Webb recipe if $p=2$, construct the graph $D$ corresponding to $G$ and let $P$ be the associated $p$-group on generators $x_{1}, x_{2}, \ldots, x_{n}$. Identifying $G$ as a subgroup of Aut $P$, let $H$ be the semidirect product $G P$.

We claim first that $Z(P)$ is characteristic in $H$. For if $R$ is any normal subgroup of exponent $p$ in $H$, then $[P, R] \leq P \cap R \leq \Omega_{1}(P)=Z(P)$ and hence, $P R / Z(P)$ is abelian. If $R \nless P$, then $P R \cap G \neq 1$, contradicting the fact that $G$ acts faithfully on $P / Z(P)$. Therefore, $R \leq P$, whence $R \leq Z(P)$. Thus, $Z(P)$ is the unique maximal normal subgroup of exponent $p$ in $H$.

If $g \in G$, there is a permutation $\pi$ of $\{1,2, \ldots, n\}$ such that

$$
x_{i}^{g} \equiv x_{\pi(i)} \quad \bmod Z(P)
$$

and so

$$
\left[x_{i}, x_{j}\right]^{g}=\left[x_{i}^{g}, x_{j}^{g}\right]=\left[x_{\pi(i)}, x_{\pi(j)}\right] .
$$

If $g \neq 1$, then $\pi(i) \neq i$ for some $i$. Since $n>2$, we may choose $j \notin\{i, \pi(i)\}$. The commutator $\left[x_{i}, x_{j}\right]$ is then not centralized by $g$. It follows from this that $C_{G}(Z(P))=1$ and thus, $P=C_{H}(Z(P))$ which is characteristic in $H$.

If $\alpha$ is any automorphism of $H$, then $\alpha$ induces by restriction an automorphism of $P$, and so for some $g \in G, \beta=\alpha i_{g}^{-1}$ acts trivially on $P / Z(P)$ (where $i_{g}$ denotes the inner automorphism corresponding to $g$ ). If $h \in H$, then for every $x \in P$,

$$
x^{h} \equiv\left(x^{h}\right)^{\beta} \equiv\left(x^{\beta}\right)^{h^{\beta}} \equiv x^{h^{\beta}} \quad \bmod Z(P)
$$

and so $h^{\beta} h^{-1} \in C_{H}(P / Z(P))=P$. Thus, $h^{\alpha} \equiv h^{g} \bmod P$ and so $\alpha$ induces on $H / P$ the inner automorphism corresponding to the element $g P$. This completes the proof of the theorem. 
The corollary is immediate, at least for the first two of the classes listed (and, of course, for some others not explicitly mentioned). The result for nilpotent $\pi$-groups is an easy consequence of the fact that such groups are direct products of their Sylow subgroups.

\section{REFERENCES}

1. H. Heineken and H. Liebeck, The occurrence of finite groups in the automorphism group of nilpotent groups of class 2, Arch. Math. 25 (1974), 8-16.

2. P. E. Schupp, A characterization of inner automorphisms, Proc. Amer. Math. Soc. 101 (1987), 226-228.

3. U. H. M. Webb, The occurrence of groups as automorphisms of nilpotent p-groups, Arch. Math. 37 (1981), 481-498.

Department of Mathematics, University of Toledo, Toledo, Ohio 43606 